\title{
Immunohistopathological Profile and Biomolecular Level of Central Nerve Tumors in Kinshasa, DRC According to the Classification of the World Health Organization 2016
}

\author{
Kisile Mikuo Olive ${ }^{1}$, Kabongo Mpolesha Jean Marie ${ }^{1}$, Claudia Manini ${ }^{2}$ \\ ${ }^{1}$ Pathological Anatomy Departmen, Medical School, Kinshasa University Clinics, Kinshasa, Democratic Republic of the Congo \\ ${ }^{2}$ Pathological Anatomy Department, Hospital Center of San Giovanni Bosco, Turin, Italy
}

Email address:

olive_kisile@yahoo.fr (K. M. Olive)

\section{To cite this article:}

Kisile Mikuo Olive, Kabongo Mpolesha Jean Marie, Claudia Manini. Immunohistopathological Profile and Biomolecular Level of Central Nerve Tumors in Kinshasa, DRC According to the Classification of the World Health Organization 2016. International Journal of Immunology. Vol. 9, No. 2, 2021, pp. 29-36. doi: 10.11648/j.iji.20210902.12

Received: March 10, 2021; Accepted: April 29, 2021; Published: May 14, 2021

\begin{abstract}
The goal is to determine immunohistochemical histopathological profile and biomolecular level of Central nerve Tumors in Kinshasa, DRC. According to the classification of the World Health Organization (WHO) 2016. Our study is described on a series of cases that were studied using standard histopathological techniques, before undergoing specific immunohistochemical techniques for the detection of anti-IDH1antibodies in gliomas and biomolecular ones in the search for $1 \mathrm{p} 19 \mathrm{q}$ deletion. Central nerve tumors account for $0.18 \%$ of all conditions, over a period of time. Meningothelial-type meningioma was the most common (60.9\%). The most affected age group is over 50 . The distribution of tumors by sex shows a predominance of woman (67.1\%). The gliomas had constituted $29.6 \%$ of all nerve tumors recorded over the period considered and whose predominance was constituted by oligoastrocytomas with $42.1 \%$. The immunohistochemical profile is characterized by a strong positivity with the anti-IDH1antibody in case of grade II oligoastrocytoma with $6.6 \%$. Our study showed that central nerve tumors account for 0.18 of all conditions, gliomas are made up of $29.6 \%$ of all nerve tumors listed with a predominance of oligoastrocytomas. The immunohistochemical profile is characterized by a strong positivity with the anti-IDH1antibody in case of grade II.
\end{abstract}

Keywords: Tumors, Glioma, Histopathologic, Immunohistochemestry, Biomolecular, Classification WHO 2016

\section{Introduction}

The gliomas are the most common primary brain tumors and group together different entities with very different prognosis. Anatomopathological examination is the standard for the diagnosis of these tumors.

However, pathologists may encounter diagnostic difficulties due, among other things to tumor heterogeneity. In this context, the confrontation between anatomopathological aspects and molecular biology are necessary.

We have recently witnessed major advances in the discovery of molecular alterations in these tumors, which led to the development of new molecular markers, some with a diagnostic role, others with a prognostic and therapeutic role $[1,2]$
A better understanding of the mechanisms of these tumors and new therapeutic avenues are essential to improve the prognosis of patients with brain tumors.

In previous studies, the classification then used for gliomas that of the World Health Organization 2007. This classification was based on the histological aspects of gliomas. However, major inter-observer discrepancies have been demonstrated for the histological diagnosis of the same tumor type and especially for the diffuse infiltranting gliomas [3].

Due to these limitations, new approaches were needed to reduce this lack of reproducibility and improve the identification of prognostic factors.

In the different studies, it was shown that the molecular classification was better correlated with the prognosis than the histological classification [4-6]. 
A new WHO classification integranting molecular markers with histological data was finally established in 2016 [1].. This classification into more homogeneous subgroups makes it possible to better guide the different therapeutic options.

According to statistical data from the World Health Organization, brain tumor pathologies correspond to the following figures: 32.6 million recorded cases, 14 million diagnosed and 8.2 million deaths annually [7]. However, despite the significant therapeutic and diagnostic arsenal, the statistics of cancerous pathologies keep showing a continuous increase, as evidenced by the figures published by the World Health Organization [7].

In Democratic Republic of the Congo, few data related to the incidence of brain tumors are known. And this is due to the lack of a cancer registry.

Therefore, we proposed to conduct a descriptive study on a series of cases relating to histopathological profile and biomolecular level of central nerve tumors of patients admitted to Pathological Anatomy departments of Kinshasa University Clinics, Leboma laboratory and the National Biomedical Research Intitute and this, over a period of 14 years from 2004 to 2018, representing a number of 64 cases.

The new classification 2016 groups together different tumor entities defined both by the histological aspect but also by the anomalies observed in immunohistochemistry and molecular biology.

It is therefore important to continue to improve knowledge on the pathophysiology of gliomas and to continue to improve the classification systems in order to better define the prognosis and especially the therapeutic management.

We are interested in coupling the histological classification of 2007 to the biomolecular classification of 2016 .

\section{Material and Methods}

\subsection{Material}

Our study spans a period of 14 years, from 2004 to 2018. It is a consecutive series of 64 cases out of a total number of 34.406 biopsies, collected from Kinshasa University Clinics, (2004-2018): 11 cases of National Institute for Biomedical Research (l'INRB) (2004-2018): 17 cases and of LEBOMA laboratory (2013-2018): 36 cases.

The paraffin slides and blocks were taken from the archives of the aforementioned establishments and reexamined respectively according to the 2007 classification and the molecular classification of the WHO 2016.

All the fragments reached us fixed in $10 \%$ formalin. After dehydration, embedding in paraffin and microtome cutting, the preparations thus obtained were stained with hematoxylin-eosin-saffron.

The data collected was recorded and analyzed using SPSS version 12 software under Windows.

\subsection{Methods}

Our study is descriptive on a series of cases, having involved 64 blocks which were first of all studied according to standard histopathological techniques (or routine), before undergoing specific immunohistochemical techniques for the search of expression of anti- IDH1 and IDH2 antibodies in gliomas and biomolecular ones in the search for the deletion of the $1 \mathrm{p} 19 \mathrm{q}$ genes.

Standard or routine histopathological Techniques: The biopsy tissue fragments fixed with $10 \%$ in formalin were dehydrated in alcohol, thinned with xylene before being impregnated in paraffin. The blocks obtained were cut with a microtome and fine ribbons of 3 to 5 microns were mounted on slides coated with albumin. After drying in an oven, these slides were stained with hematoxylin-Eosin before mounting a coverslip fixed by l'Eukit. In fact, after comes the optical microscope reading for the typing of our nerve tumors. The immunohistochemical study included the anti-IDH1 monoclonal antibody (clone H09), by resorting to Benchmark Ultra (Ventana-Roche), who is an immunohistochemistry automaton and according to the following steps: The tissue blocks fixed with $10 \%$ formalin and embedded in paraffin are cut with a microtome. The thin 4 micron ribbons were placed on the polysilane slides and dried in the oven for at least 12 hours at 37 degrees. We will then incubate the primary and secondary antibody then the developer according to the following steps:

1) Dewaxing with two xylene baths of 10 minutes each followed by rehydration with absolute alcohol ( 2 X 5 minutes) and a 5 minutes alcohol bath) 80 degrees then distilled water.

2) Pretreatment in a water bath heated to 96 degrees and in the buffer recommanded by the manufacturer (EDTA or Citrate) for 30 to 40 minutes depending on the antigen to be unmasked.

3) After cooling for 20 minutes, circle the preparations with Dako-pen (Hydrophobic marker) then place 3\% hydrogen peroxide for 20 minutes. Rinse the slides with distilled water and then in Tris-Buffer-Salin (TBS).

4) Incubation with the primary antibody (diluted according to the manufacturer's recommandations: 30 60 minutes depending on the antibody).

5) Rinse in distilled water then TBS

6) Incubation with the secondary antibody (30 minutes).

7) Rinsing as above.

8) Place the developer (DAB:Di-amino-3.5 Benzidine or AEC: Amino-9 Ethyl-Carbazole) for 5 minutes.

9) Rinsing with normal water.

10)Do a counterstain with Hemalun of Mayer (aqueous solution).

11)Rinsing as above.

12)Assembly of the coverslips with aqueous adhesive (Paramount or Pertex or Eukit)

13)Optical microscope reading.

14)Molecular analyzes, in particular the $1 \mathrm{p} 19 \mathrm{q}$ deletions are in progress and the results will be published later. Fug. 16 shows the different characteristics of the antiIDH1 R132H antibody, clone H09: 

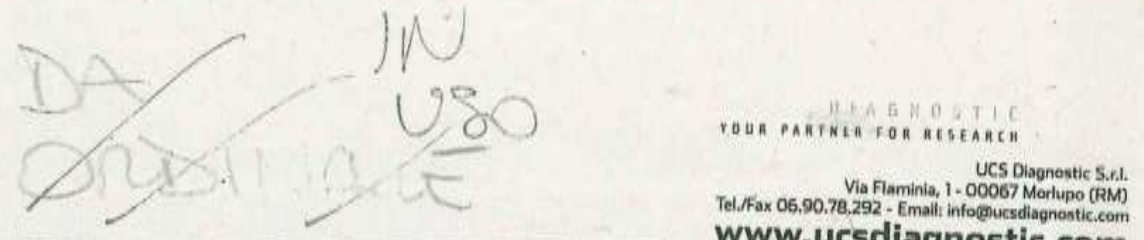

Tel./Fax 06,90,78,292 - Emailt info(mucsdiagnontic.corm

WWW.ucsdiagnostic.com

\section{Anti-IDH1 R132H / DIA-H09 Mouse monoclonal anti-brain tumor marker (Astrocytoma, Oligodendroglioma), Clone H09}

\section{Product Information}

\begin{tabular}{|c|c|}
\hline Catalog No.: & 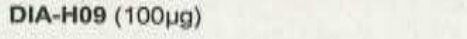 \\
\hline Clone: & $\mathrm{H} 09$ \\
\hline Concentration: & $0.2 \mathrm{mg} / \mathrm{ml}$ \\
\hline Isotype: & Mouse $\lg \mathrm{G}_{2 \mathrm{a}}$ \\
\hline Specificity: & Human IDH1 R132H point mutation \\
\hline Immunogen: & $\begin{array}{l}\text { Synthetic peptide, amino acid sequence } \\
\text { CKPIIIGHHAYGD }\end{array}$ \\
\hline $\begin{array}{l}\text { Physical State: } \\
\text { Species }\end{array}$ & Lyophilized powder \\
\hline $\begin{array}{l}\text { Reactivity: } \\
\text { Positive }\end{array}$ & Human \\
\hline $\begin{array}{l}\text { Control: } \\
\text { Negative }\end{array}$ & Oligodendroglioma, diffuse astrocytoma \\
\hline Control: & $\begin{array}{l}\text { Pilocytic astrocytoma, primary glioblasto- } \\
\text { ma (ca. } 95 \% \text { of cases negative) }\end{array}$ \\
\hline Visualization: & Cytoplasmic \\
\hline
\end{tabular}

Reconstitution: DIA-H09 $(100 \mu \mathrm{g})$, restore to $500 \mu \mathrm{I}$ Reconstitute with sterile distilled water

Presentation: In PBS with $2 \%$ BSA, $0.05 \%$ NaN3, by gentle shaking for 10 minutes $\mathrm{pH}$ 7.4. Antibody purified from culture supernatant

Applications: Immunohistochemistry

(standard formalin-fixed paraffin sections)

Dilutions:

Western blot

1:20 Immunohistochemistry (IHC)

1:500 Western Blot

(General recommendation, validation of antibody performance/protocol is the responsibility of the end user. Positive/negative controls should be run simultaneously with patient spocimen. Interpretation must be made by a qualified pathologist within the context of patient's clinical history/other diagnostic tests.)

Control Antibody: DIA-W09, rat anti-hu IDH1wt, clone W09

(Staining protocols on www.dianova.com)

\section{Reactivity}

Antibody clone $\mathrm{H} 09$ reacts specifically with the isocitrate dehydrogenase 1 (IDH1) R132H point mutation in tissue sections from formalin-fixed brain tumor specimens. Heterozygous point mutations of IDH1 codon 132 are frequent in World Health Organization (WHO) grade II and III gliomas. IDH1 R132H mutations occur in approximately $70 \%$ of astrocytomas and oligodendroglia tumors. The high frequency and distribution of the IDH1 R132H mutation among specific brain tumor entities allow the highly sensitive and specific discrimination of various tumors by immunohistochemistry, such as anaplastic astrocytoma from primary glioblastoma or diffuse astrocytoma WHO grade II from pilocytic astrocytoma or ependymoma. Noteworthy is the discrimination of the infiltrating edge of tumors with IDH1 mutation from reactive gliosis. This antibody is highly useful for tumor classification and in detecting single infiltrating tumor cells.

\section{Instructions for Use}

Immunohistochemical staining of standard formalin-fixed paraffin sections

Deparaffinize and rehydrate according to standard procedures. Heat induced epitope retrieval (HIER) is required. For immunohistochemical detection different techniques can be used: Indirect immunoenzyme labeling with a secondary antibody conjugate, biotin/(strept)avidin-based detection, soluble enzyme immune complex or polymer-based detection. To detect antibody, follow the instructions provided with the particular visualization system. The antibody is suited for immuno-histochemical staining using automated platforms. Use the antibody at 1:20 dilution for $30 \mathrm{~min}$ at RT.

Technical note

Diffuse astrocytoma WHO grade II may have low protein-expression. At high dilution of the antibody single tumor cells in the infiltration zone may not be stained.

Intented use / regulatory status

Europe: For in Vitro Diagnostic Use / All other countries: For Research Use only

\section{Storage and Stability}

Store the lyophilized antibody at $2-8^{\circ} \mathrm{C}$. For long time storage freeze at $-20^{\circ} \mathrm{C}$, thus the antibody is stable for at least one year. As reconstituted liquid store at $2-8^{\circ} \mathrm{C}$ short term (several weeks). For long term storage aliquot and freeze at $-20^{\circ} \mathrm{C}$ or $-80^{\circ} \mathrm{C}$. Avoid repeated freeze / thaw cycles.

\section{Safety Notes}

The material contains $0.05 \%$ sodium azide as preservative. Although the quantity of azide is very small, appropriate care should be taken when handling this material. Avoid skin and eye contact, inhalation, and ingestion.

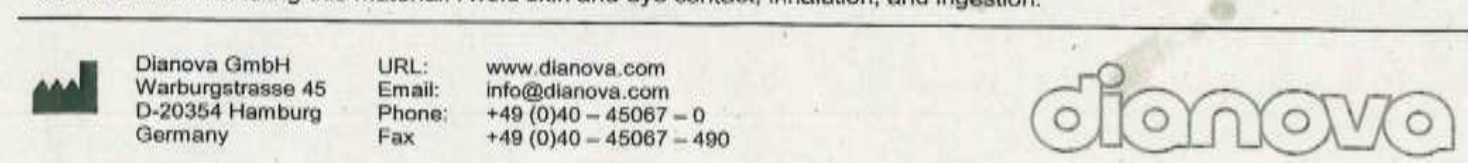




\section{Figures}

Immunohistochemistry of human IDH1 R132H in formalin-fixed paraffin-embedded brain tissue sections (pictures courtesy of Prof. Dr. med. Andreas von Deimling, Department of Neuropathology, University Heidelberg / Clinical Cooperation Unit Neuropathology, German Cancer Research Center (DKFZ), Heidelberg, Germany)

A: Strong reaction of IDH1 mutation specific antibody clone $\mathrm{HO}$ in tumor center of anaplastic oligoastrocytoma.

B: Infiltration zone of anaplastic astrocytoma with specific labelling of infiltrating glioma cells by antibody clone $\mathrm{H} 09$

C: Identification of single tumor cells in white matter distant from tumor center with IDH1 mutation specific antibody clone $\mathrm{HOS}$.

D: Cortex infiltrated by oligodendroglioma with specific labelling of tumor cells by antibody clone $\mathrm{H} 09$.

E: Double staining of GFAP (glial fibrillary acidic protein, red) and clone HO9 (brown) of oligodendroglioma infiltration zone demonstrating specific labelling of tumor cells but not of GFAP positive reactive astrocytes.

F: Strong reaction of IDH1 mutation specific antibody clone $\mathrm{H} 09$ with IDH1 R132H mutated diffuse astrocytoma (left) but not with wild type tumor (right).
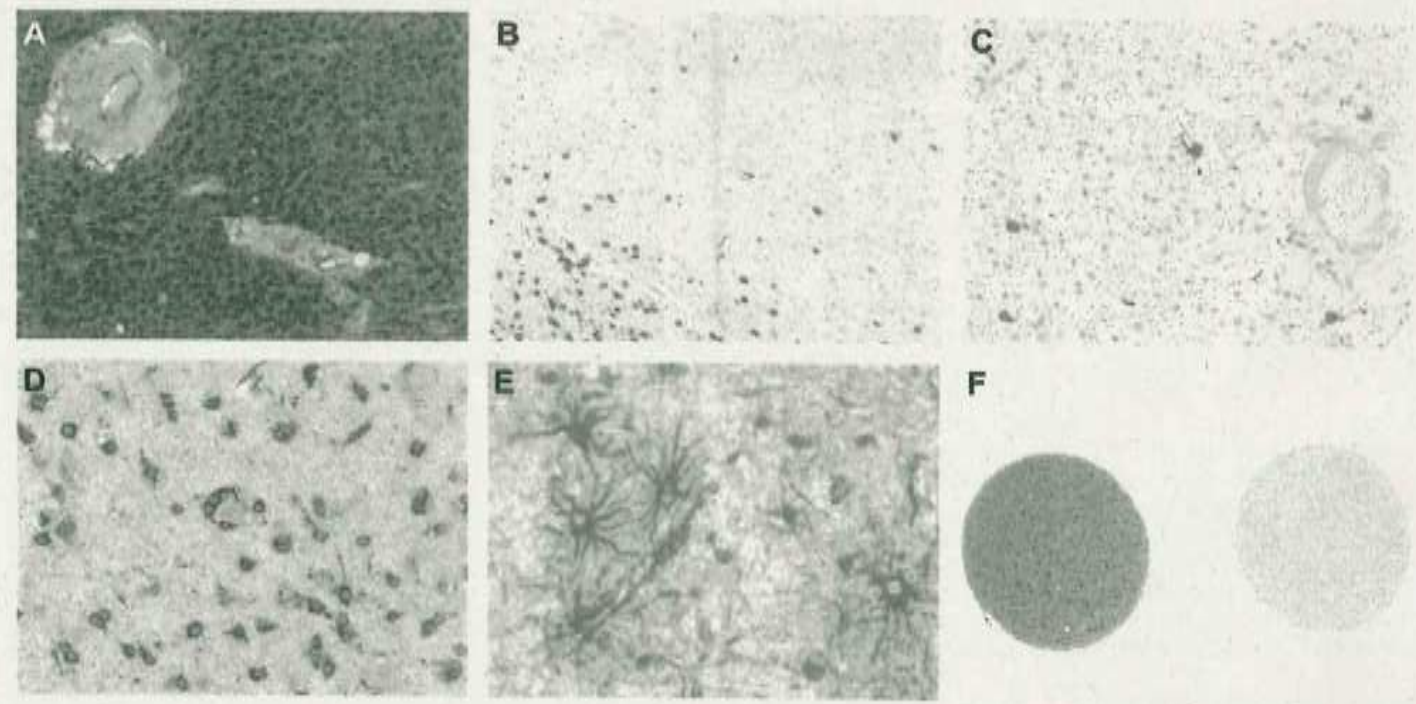

F

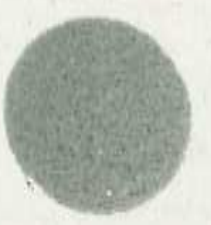

\section{References}

1. Capper D et al. Monoclonal antibody specific for IDH1 R132H mutation. Acta Nouropathol. 118(5): 599-601, 2009

2. Capper D et al. Characterization of $\mathrm{R} 132 \mathrm{H}$ mutation-specific IDH1 antibody binding in brain tumors. Brain Pathol. 20(1): $245-254,2010$

3. Preusser $\mathrm{M}$ et al. IDH testing in diagnostic neuropathology: review and practical guideline article invited by the Euro-CNS research committee. Clinical Neuropathology, 30(5):217-230, 2011

4. Van den Bent MJ et al, Interlaboratory comparison of IDH mutation detection. J Neurooncol 112:173-178, 2013

5. Schumacher $\mathrm{T}$ et al. A vaccine targeting mutant IDH1 induces antitumour immunity. Nature 2014, DOI:10.1038/nature13387

6. David NL et al. The 2016 World Health Organization Classification of Tumors of the Central Nervous System: a summary. Acta Neuropathol., 131:803-820, 2016

\section{Symbols}

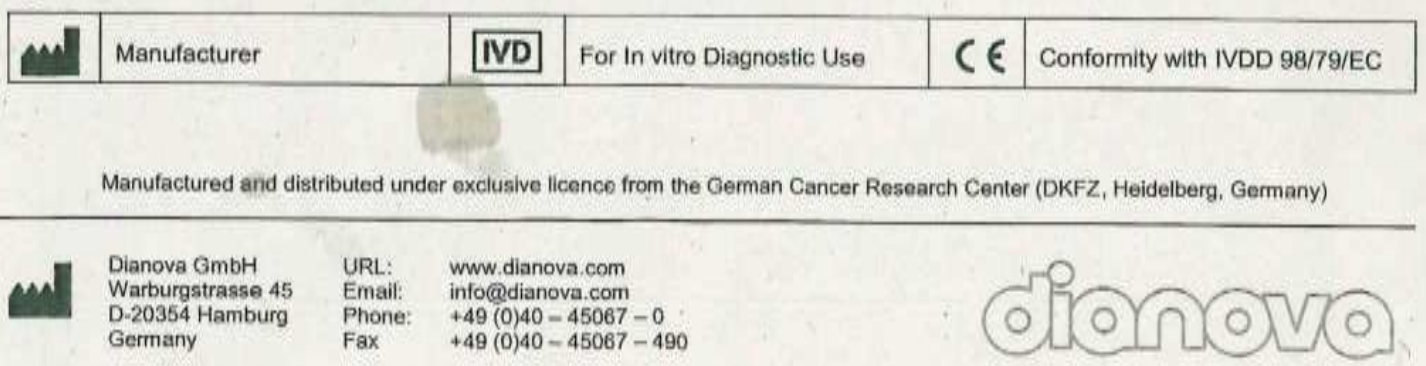

Figure 1. Characteristics of anti-IDH1 antibody. 


\section{Results}

\subsection{Relative frequency of Central Nerve Tumors}

During the period from 2004 to 2018,34406 biopsies were recorded, including 64 cases of central nerve tumors, i.e a frequency of $0.18 \%(64 / 34406)$.

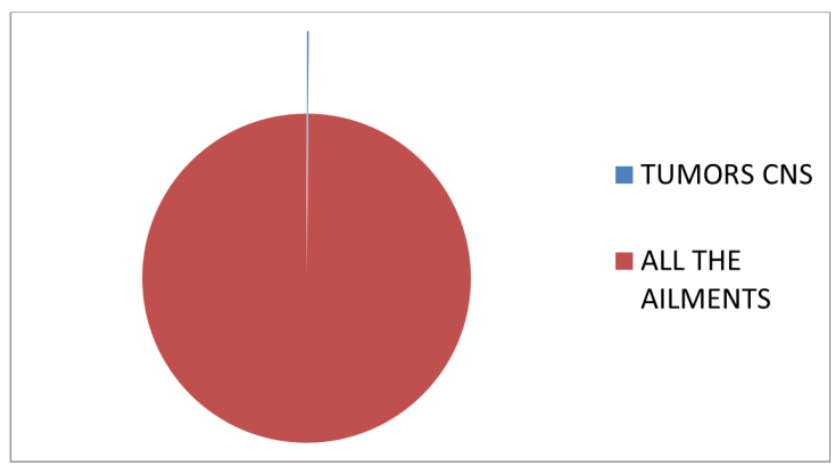

Figure 2. Frequency of central nerve tumors.

\subsection{Relative Frequency of Different Histopathological Types of NC Tumors}

Table 1. Relative frequency of histopathological types of nerve central tumors.

\begin{tabular}{lll}
\hline Histopathological Types & Number & Frequency (\%) \\
\hline Meningiomas & 39 & 60.9 \\
Oligoastrocytomas & 8 & 12.5 \\
Astrocytomas & 5 & 7.8 \\
Oligodendrogliomas & 3 & 4.6 \\
Glioblastomas & 3 & 4.6 \\
Ependymomas & 3 & 4.6 \\
Retinoblastomas & 2 & 3.1 \\
Choroid plexus papilloma & 1 & 1.50 \\
Total & 64 & 100 \\
\hline
\end{tabular}

The table above shows that meningioma comes first with $60.9 \%$ of cases followed by oligoastrocytomas.

\subsubsection{Frequency of Different Histopathological Types of Meningiomas}

Table 2. Frequency of different histopathological types of meningiomas.

\begin{tabular}{lll}
\hline Histopathological Types & Number & Frequency (\%) \\
\hline Meningothelial Meningioma & 22 & 56.4 \\
Anaplastic Meningioma & 5 & 12.8 \\
Psammomatous Meningioma & 4 & 10.2 \\
Mixed Meningioma & 3 & 7.6 \\
Fibroblastic Meningioma & 2 & 5.1 \\
Papillary Meningioma & 2 & 5.1 \\
Angiomatous Meningioma & 1 & 2.5 \\
Total & 39 & 100 \\
\hline
\end{tabular}

The table above shows that the meningothelial type meningioma is the most frequent with $56.4 \%$.

\subsubsection{Frequency of Different Histopathological Types of Astrocytomas}

Table 3. Frequency of different histopathological types of astrocytomas.

\begin{tabular}{lll}
\hline Histopathological Types & Number & Frequency (\%) \\
\hline Oligoastrocytoma & & \\
Diffuse Gemistocytic & 8 & 50 \\
Astrocytoma & 3 & 18.7 \\
Glioblastoma & 3 & 18.7 \\
Diffuse fibrillar Astrocytoma & 2 & 12.5 \\
Total & 16 & 100 \\
\hline
\end{tabular}

The table above shows a predominance of oligoastrocytomas with $50 \%$.

\subsection{Distribution of Tumors by Sex}

The distribution of tumors by sex shows a predominance of woman with $67.1 \%(43 / 64)$ and men: $32.8 \%(21 / 64)$. The sex ratio is 0.48 .

\subsection{Distribution of Tumors According to Different Age Groups}

Table 4. Distribution of tumors according to different age groups.

\begin{tabular}{lll}
\hline Age groups & Number & Frequency (\%) \\
\hline $1-10$ & 4 & 6.20 \\
$11-20$ & 4 & 6.20 \\
$21-30$ & 1 & 1.50 \\
$31-40$ & 7 & 10.90 \\
$41-50$ & 16 & 25 \\
Sup. 50 & 32 & 50 \\
Total & 64 & 100 \\
\hline
\end{tabular}

The table above shows that the age groupmost affected by central nerve tumors was over 50 with $50 \%$, followed by 41 50 with $25 \%$. The youngest patient was 20 months old with retinoblastoma and the oldest with malignant meningioma was 75 years old.

\subsection{Frequency of Different Histopathological Types of Gliomas}

Table 5. Frequency of different histopathological types of gliomas.

\begin{tabular}{lll}
\hline Histopathological Types & Number & Frequency (\%) \\
\hline Oligoastrocytomas & 8 & 42.1 \\
Astrocytomas & 5 & 26.3 \\
Glioblastomas & 3 & 15.7 \\
Oligodendrogliomas & 3 & 15.7 \\
Total & 19 & 100 \\
\hline
\end{tabular}

The table above shows that gliomas constituted $29.6 \%$ of all central nerve tumors listed over the period of considered and the predominance of which was constituted by oligoastrocytomas with $42.1 \%$. 


\subsection{Frequency of Different Histopathological Types of Gliomas by Sex}

Table 6. Frequency of different histopathological types of gliomas by sex.

\begin{tabular}{llllll}
\hline Tumors & Number & Feminine & $\begin{array}{l}\text { Frequency } \\
(\mathbf{\%})\end{array}$ & Male & $\begin{array}{l}\text { Frequency } \\
(\mathbf{\%})\end{array}$ \\
\hline Oligoastrocytomas & 7 & 6 & 31.5 & 1 & 5.2 \\
Oligodendrogliomas & 3 & 1 & 5.2 & 2 & 10.5 \\
Astrocytomas & 6 & 4 & 21 & 2 & 10.5 \\
Glioblastomas & 3 & 2 & 10.5 & 1 & 5.2 \\
Total & 19 & 13 & 68.4 & 6 & 31.5 \\
\hline
\end{tabular}

The frequency of different histopathological types of gliomas by sex, as shown in the table above, shows a predominance of women.

\subsection{Immunohistochemical and Molecular Profile of Gliomas According to the World Health Organization Classification 2016}

Table 7. Expression $\mathrm{OF} I D H 1$ in gliomas.

\begin{tabular}{ll}
\hline Gliomas & IDH 1 \\
\hline Polymorphic Glioblastoma & Negative \\
Anaplastic Oligoastrocytoma & Negative \\
grade I Oligodendroglioma & Negative \\
Giant Cell Glioblastoma & Negative \\
Grade II Oligoastrocytoma & ++ \\
Grade II Oligoastrocytoma & Negative \\
Grade II gemistocytic Astrocytoma & Negative \\
Grade II Oligoastrocytoma & Negative \\
Oligoastrocytoma & Negative \\
Diffuse gemistocytic Astrocytoma & Negative \\
Grade II Oligoastrocytoma & ++++ \\
Grade II Oligoastrocytoma & Negative \\
Grade II gemistocytic Astrocytoma & Negative \\
Grade II Oligodendroglioma & ++ \\
Anaplastic Astrocytoma & Negative \\
\hline
\end{tabular}

This table illustrates the expression of IDH1 in gliomas.

This expression is characterized by a strong positivity with the anti-IDH1 antibody in a case of grade II oligoastrocytoma in the 15 cases of glioma, i.e. $6,6 \%$ (Figure 3 ), low positivity in $2 / 15$ (i.e. $13.3 \%$ ) cases: 1 case of grade II oligodendroglioma and a grade II oligoastrocytoma case (Figure 4) and IDH1 negative in 12 cases.

This figure illustrates a strongly positive IDH1 oligoastrocytoma

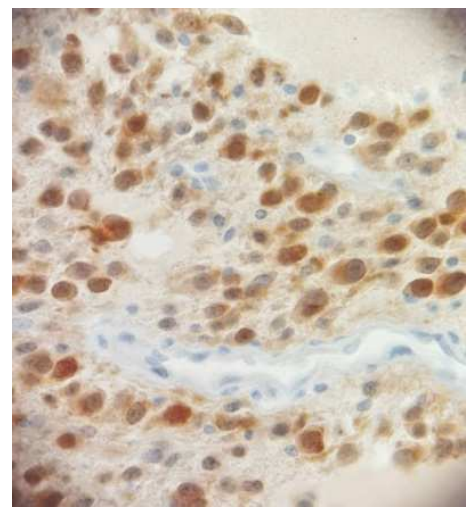

Figure 3. Microscopic image of a strongly positive IDH1 oligoastrocytoma.
This figure illustrates a strongly positive IDH1 oligoastrocytoma

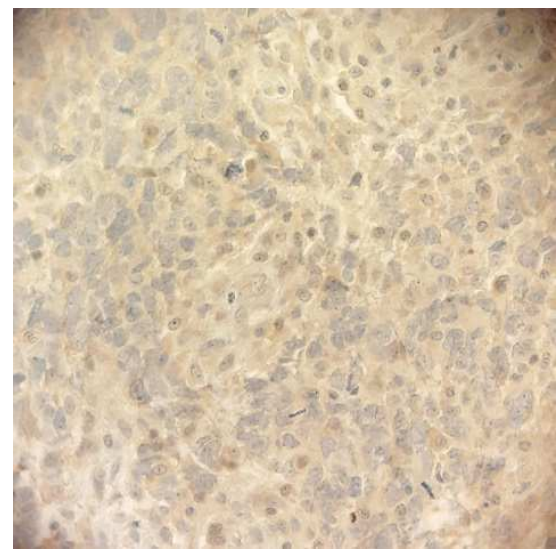

Figure 4. Microscopic image of a weak positive IDH1 oligoastrocytoma.

This figure illustrates a strongly positive IDH1 oligoastrocytoma

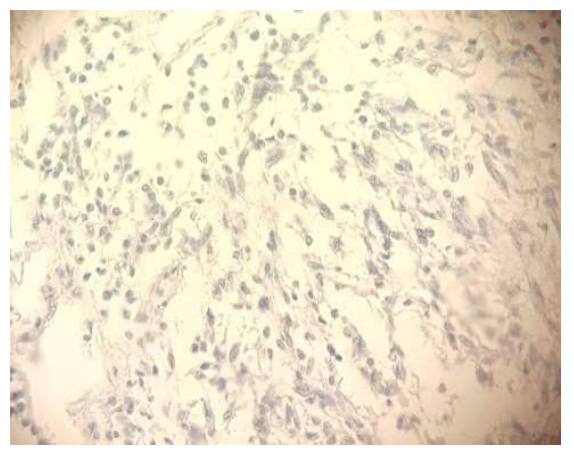

Figure 5. Microscopic image of an IDH1 negative astrocytoma.

\section{Discussion}

\subsection{Frequency Profile of Intracranial Tumors}

In our study, the frequency of central nervous system tumors (SNC) in the aforementioned institutions was $0.18 \%$, on a sample of 34,406 biopsies, during 14 years (2004-2018).

Eyenga VC and coll. reported $6.3 \%$ and $5 \%$ respectively [8]. The annual incidence rate in France between 1980 and 2005 is 5.7 per 100,000 in men and de 4.2 per 100,000 in women [9].

Compared to the overall number of recorded cases, our results are lower than western data [10]. and approximate the data of the Maghreb countries [11].

The variations in frequencies can be explained by the absence in our country of a national cancer registry, consequently of a database; the disparity of the healthcare system, the size of the populations and accessibility to healthcare.

In our study, women represented $67.1 \%$ against $32.8 \%$ for men. Male predominance is reported in most of national series [12], maghrebian [13]. Congolese [14]. in our previous and international study [15]. This difference could be associated with the size of our sample. In our series, the age 
group over 50 is the most affected, our results are similar to most of the series reported in the literature [7].

Gliomas had a frequency of $29.6 \%$, this result is contrary to that observed in other studies $[15,16]$.

The preliminary glioma analysis was carried out on 64 patients in the three aforementioned laboratories. The median age of our patients is 30 years with an age range of 20 months to 59 years. This average seems to be much lower compared to the world average given by the WHO which is 53 years old and the European average which is 50 years according to the WHO [7].

However, the average age that we obtained turns out to be close to that observed in Moroccan statistics, reported by the study of EL MADHI and coll. [15]. which is 35 years old. This could correlate with a genetic predisposition and an environmental component. Indeed, if the TAO and coll study [17]. affirms that brain tumors occur more commonly in humans, regardless of age and geographic location, it also gives a sex ratio $\mathrm{M} / \mathrm{F}$ of 1.3 . Our study, for its part, reports the opposite with a sex-ratio $\mathrm{M} / \mathrm{F}$ of 0.48 . This further supports the involvement of a genetic and environmental.

\subsection{Immunohistochemical and Molecular Profile of Gliomas According to the WHO Classification 2016}

One of the objectives of this study was to characterize from an immunohistochemical point of view, gliomas in the light of the WHO classification 2016. In this first part we were able to carry out the search for anti IDH1 antigens. In a subsequent study, the second part will be carried out which will consist in the practice of biomolecular analyzes. (codeletion 1p19q fusion KIAA1549-BRAF, ....)

The $\mathrm{R} 132 \mathrm{H}$ mutation of IDH1 is present in $13.3 \%$ of grade II oligoastrocytoma and $6.6 \%$ oligodendrogliomas II. IDH1 mutations are common in grade II and III invasive gliomas $[17,18]$, associated with TP53 mutations in diffuse astrocytomas [19]. These mutations are involved in early gliomagenesis. However, the IDH1 mutations usually reported are between 50 and $70 \%$ of oligodendrogliomas [20, 22], against $7.4 \%$ in our series. Our results confirm that it is essential to determine IDH1 status in routine diagnosis and reinforces the hypothesis that the same tumor stem cell is at the origin of the different contingents of the same tumor but that the distinct oncogenic mechanisms are at the origin of infiltrating and non-infiltrating gliomas.

The expression of IDH1 is linked to the presence of an oligodendroglial contingent in an infiltrating glioma but not to that of the contingent «oligo-like» in non-infitrating tumors.

The analysis of survival data is consistent with the literature. In our serie, the cases for which the expression of IDH1 is positive, have a better prognosis. Non-infiltrating tumors (Pilocytics astrocytomas) have a better prognosis than invasive gliomas. Among the latter, les oligodendrogliomas have a favorable evolution than oligo-astrocytomas and glioblastomas. In oligodendrogliomas, the presence of an IDH mutation correlates with better recurrence-free survival, which is consistent with known.

The many negative anti-IDH1 cases, with a poor prognosis, could be explained by the fact that patients consult late, when the tumor has already progressed sufficiently in grade.

\section{Conclusion}

This study revealed a frequency of $0.18 \%$ of central nerve tumors, over a period of 14 years, a lower frequency compared to other studies, both national and international.

Meningioma comes first, followed by oligoastrocytomas. Meningothelial-type meningioma is the most common.

A predominance of diffuse gemistocytic astrocytomas and glioblastomas was noted.

The distribution of our series by sex showed a predominance of women.

The age group most affected by central nerve tumors is 41 50 years old.

Gliomas had constituted $29.6 \%$ of all central nerve tumors recorded over the period considered and whose predominance is constituted by oligoastrocytoma.

They were more concerned with the age groups above 50 years and the female sex.

The immuno-molecular study of the different tumor cell contingents of non-infiltranting gliomas in comparison with oligodendrogliomas, indicates that morphologically identical contigents have a different molecular profile. The oncogenesis of non-infiltrating gliomas is quite non different from that of invasive gliomas (oligodendrogliomas). Infiltranting and non- infiltranting gliomas, therefore do not derive from the same tumor stem cell.

We note here a predominance of negative cases for antiIDH1 antibodies.

The immunohistochemical study is useful for the differential diagnosis between non invasive pilocytic astrocytoma and oligodendroglioma using anti- IDH1 antibodies.

Our results encourage us to continue this study by expanding our sampling and exploring all the biomolecular analyzes that are in progress.

\section{Conflicts of Interest}

The authors declare no conflict of Interest.

\section{References}

[1] DN LOUIS, WIESTLER OD, and CAVENEE WK. WHO classification of tumors of the central nervous system. Revised $4^{\text {th }}$ edition, Lyon: International Agency for Research on Cancer (IARC) 2016.

[2] WELlER M, STUPP R, HEGI ME and coll. Personalized care in neuro-oncology coming of age: why we need MGMT and $1 \mathrm{p} / 19 \mathrm{q}$ testing for malignant glioma patients in clinical practice. Neuro Oncol 2012; Suppl. 4: iv100-108. 
[3] VAN DEN BENT MJ. Interobserver variation of the histopathological diagnosis in clinical trials on glioma: a clinician's perspective. Acta Neuropathol. 2010 Sept; 1205: 297-304.

[4] BRAT DJ, VERHAAK RG, ALDAPE KD and coll. Cancer Genome Atlas Research Network, Comprehensive, Integrative Genomic Analysis of diffuse Lower-Grade Gliomas. $N$ Engl J Med. Jun 25; 372 (26): 2481-2498.

[5] SUZUKI H, AOKI K, SATO Y and coll. Mutational landscape and clonal architecture in grade II and III gliomas. Nat Genet. 2015 May; 47(5): 458-468,

[6] WIESTLER B, CAPPER D, SILL M and coll. Integrated DNA methylation and copy-number profiling identify three clinically and biologically relevant groups of anaplastic glioma. Acta Neuropathology 2014 Oct; 128 (4): 561-571.

[7] http://globcan.iarc.fr/Lyon,10.1186/2042-6410-3-3

[8] KEITA AD, KANE M, GUINTO CO and coll. Contribution of tomodensitometry in the management of brain tumorat the $\mathrm{G}$ point HOSPITAL in Mali. Médical Mali 2007; 22 (2): 14-18.

[9] BELOT A, VELTEN M, GROSCLAUDE P et al. National estimate of cancer in incidence and mortality in France between 1980 and 2005. Saint-Maurice (Fra):French institute for public Health Surveillance, décember 2008, p132.

[10] BELOT A and coll. Cancer incidence and mortality in France over the period: 1980-2005. Epidemiology and Public Health Review, 2008, 56:159-175.

[11] FOUATIH Z and coll. The cancer registry of Oran. Ten years of registration: 1996-2005. Oran, University of Oran, Faculty of Medecine, 2008.

[12] Rabat Cancer Registry. Incidence of cancer in Rabat-2005, Rabat, Health Ministry, epidemiology and disease control department (DELM) Scientific Association of the National Institute of Oncology (ASINO), 2009.

[13] SELLAMI A and coll. Southern Tunisian Cancer Registry. Cancer Incidence 1997-19998.
[14] KISSILE O., KALENGAYI MM, PASQUIER B and coll. Anatomoclinical and epidemiological Profile of nerve tumors in congolese. Pratical Carcinology in Africa 2005; 6: 43-46.

[15] EL MADHI, T. EL AZZOUZI M, EL KHAMLICHIL A. Descriptive epidémiological profile of central nervous system tumors: About 903 case (1983-1992). Maghreb Medicine $\mathrm{N}^{\circ} 59$.

[16] TAO S, WARRINGTON NM, RUBIN JB. Why does Jack and not Jill, break his crown? Sex disparity in brains tumors. Biology of sex differences, 3. (2012).

[17] LONJON M, MONDO L, LONJON N and coll. Clinical Pathways of glioblastomas and neuroradiology. Neurosurgery 2010; 56: 449-454.

[18] FRENEL JS, BOTTI M, LOUSSOUARN D and coll. Pronostic and Predictive factors of response in adult cerebral gliomas Bull Cancer 2009; 96 (4): 357-367.

[19] BIRNER P, TOUMANGELOVA-UZEIR K, NATCHEV S and coll. Expression of mutated isocitrate dehydrogenase-1 in glioma is associated with P53 and EGFR expression. Folia Neuropathology. 2011; 49(2):88-93.

[20] 2MELLAI M, PIAZZI A, CALDERA V and coll. IDH1 and IDH2 mutations, immunohistochemistry and associations in a series of brains tumors. $J$ Neuropathol. 2011; 105 (2): 345357.

[21] JEUKEN J, CORNELISSEN S, BOOTS-SPRENGER S and coll. Multiplex ligation-dependent probe amplification: a diagnostic tool for simultaneous identification of different genetic markers in glials tumors. J Mol Diagn 2006; 8 (4): 4439 .

[22] MUKASA A, TAKAYANAGI S, SAITO $\mathrm{K}$ and coll. Significancy IDH mutations varies with tumor histology, grade, and genetics in Japanese glioma patients, Cancer Sci. 2012; 103 (3): 587-592. 